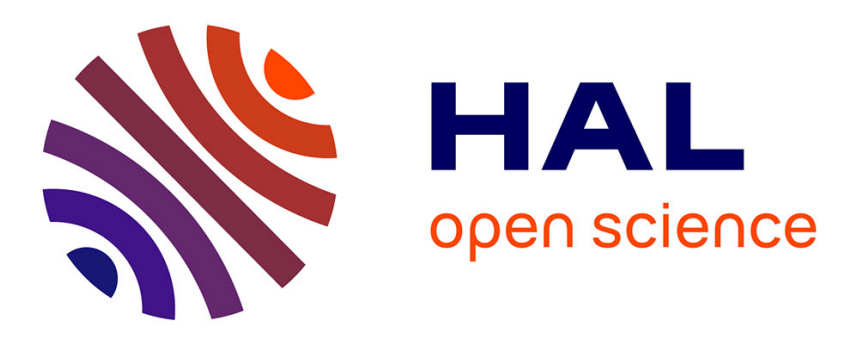

\title{
A decoupled multiple model approach for state estimation of nonlinear systems subject to delayed measurements
}

\author{
Rodolfo Orjuela, Benoît Marx, José Ragot, Didier Maquin
}

\section{- To cite this version:}

Rodolfo Orjuela, Benoît Marx, José Ragot, Didier Maquin. A decoupled multiple model approach for state estimation of nonlinear systems subject to delayed measurements. 3rd IFAC Workshop on Advanced Fuzzy and Neural Control, AFNC 2007, Oct 2007, Valenciennes, France. pp.CDROM. hal-00178139

\author{
HAL Id: hal-00178139 \\ https://hal.science/hal-00178139
}

Submitted on 27 Mar 2014

HAL is a multi-disciplinary open access archive for the deposit and dissemination of scientific research documents, whether they are published or not. The documents may come from teaching and research institutions in France or abroad, or from public or private research centers.
L'archive ouverte pluridisciplinaire HAL, est destinée au dépôt et à la diffusion de documents scientifiques de niveau recherche, publiés ou non, émanant des établissements d'enseignement et de recherche français ou étrangers, des laboratoires publics ou privés. 


\title{
A DECOUPLED MULTIPLE MODEL APPROACH FOR STATE ESTIMATION OF NONLINEAR SYSTEMS SUBJECT TO DELAYED MEASUREMENTS
}

\author{
Rodolfo Orjuela, Benoît Marx, José Ragot \\ and Didier Maquin
}

\author{
Centre de Recherche en Automatique de Nancy \\ UMR 7039, Nancy-Université, CNRS \\ 2, Avenue de la Forêt de Haye \\ 54516 Vandouvre-lès-Nancy, France
}

\begin{abstract}
The state estimation of nonlinear systems with delayed measurements is investigated in this paper. The proposed approach is based on the representation of the nonlinear system by a decoupled multiple model that, to our knowledge, has not been investigated extensively. This multiple model approach offers an interesting alternative to the classically used multiple model known as TakagiSugeno multiple model. Indeed, in contrast to this last, the decoupled multiple model makes it possible to introduce a state vector with a different dimension for each submodel. Sufficient conditions for ensuring the exponential convergence of the estimation error are provided in terms of LMIs. Copyright (c) 2007 IFAC
\end{abstract}

Keywords: decoupled multiple model, state estimation, time-delay systems, delayed measurements, nonlinear systems.

\section{INTRODUCTION}

Strongly nonlinear systems subject to delayed measurements are frequently encountered in practical engineering problems. Indeed, any measurement system has intrinsically a time-delay more or less negligible, for example due to data transmission time between the sensors of the system and the monitoring room.

The time-delay is not often taken into account in the modelling stage of the system in order to provide a less complex model useful for designing a control law or setting up a diagnosis strategy. However, it is well known that the influence of the time-delay can be at the origin of serious consequences (ignored data, oscillations, destabilization of the closed-loop, etc.) for the safety of the operator and/or to keep the system in good working order (see for example (Richard, 2003) and the references therein for an overview of delayed systems).

The state estimation of the system, using an observer, may be a way to partly avoid these kinds of problems. The state estimation problem for linear systems, with delay in the input, the output and/or the state has been widely investigated in the last few years (see for instance (Yang and Saif, 1998; Sename, 2001; Fridman et al., 2003) and their references). The resulting observer can be used in an observer-based controller perspective in order to ensure the stability of the feedback control law. In other respects, Germani et al. (2002) and Kazantzis and Wright (2005) propose a similar solution to the state estimation of a class of nonlinear systems. The two solutions are conceptually similar, the basic idea being the 
building of a chain observer that allows the state estimation at a given fraction of the output delay.

Our approach, in order to cope with the state estimation problem, is based on the multiple model approach. The nonlinear system modelling is then performed with the help of a multiple model which is closely related to operating regimebased modelling framework (Murray-Smith and Johansen, 1997). Indeed, multiple models are a powerful tool for modelling a large class of nonlinear systems. The basis of the multiple model approach is the decomposition of the operating space of the nonlinear system into a number $L$ of operating zones, each of them being characterised by a submodel. According to the zone where the nonlinear system evolves, the output of each submodel is more or less requested in order to approximate the whole behaviour of the nonlinear system. The contribution of each submodel is defined by a weighting function.

It is important to notice that a large class of complex systems can be approximated by a multiple model, with a wished accuracy, by increasing the number of submodels and by a parametric optimisation of the weighting functions. Furthermore, if the submodels take a linear form and for particular weighting functions, most of the analysis tools available for linear systems can be partially extended to the analysis of nonlinear systems represented by a multiple model.

Filev (1991) provides, in a fuzzy modelling framework (directly related to multiple model framework), two possible structures in order to take into account the contribution of each submodel. In the first structure, the submodels are decoupled and their state vector are different (decoupled multiple model); in the second one, the submodels share the same state vector (Takagi-Sugeno multiple model).

The Takagi-Sugeno model has been successfully used for modelling the dynamic behaviour of delayed nonlinear systems. Cao and Frank (2001) present the conception of a stabilization law using an observer by taking into account multiple known time-delays in the state and the output. Chen et al. (2004) establish sufficient conditions for ensuring the stability of a multiple model that represents a nonlinear interconnected system (largescale system) subject to time-delay in the state. Jiang et al. (2005) propose an observer-based feedback controller with adaptation to delay, hence the exact knowledge of time-delay is unnecessary. The time-delay considered is constant but is not known exactly. In this approach, both estimation of the time-delay and the state are achieved in the same time.
By comparison with the Takagi-Sugeno multiple model, the decoupled multiple model has been less investigated. However, a few works in the control domain (Gawthrop, 1995; Gregorcic and Lightbody, 2000) and in modelling (Venkat et al., 2003; Orjuela et al., 2006) of nonlinear systems have made a successful implementation of this structure and shown its relevance.

In this communication, a new method for designing an observer for a nonlinear system (modelled by a decoupled multiple model) subject to variable time-delay measurements is proposed. The outline of this paper is as follows. The two classic structures of a multiple model are shown in section 2. The problem formulation is presented in section 3. In section 4, the stability of the decoupled multiple model is investigated on one hand, and sufficient conditions (in LMIs terms) in order to ensure the exponential convergence of the estimation error are established on the other hand. In section 5, an academic example illustrates the state estimation of a decoupled multiple model.

\section{MULTIPLE MODEL STRUCTURES}

The interconnection of the submodels can be performed with various structures in order to generate the global output of the multiple model. Two essential structures of multiple models can be distinguished whether the same state vector appears in all submodels or not.

Concerning the identification step, there exists different techniques (linearisation, parametric optimisation) for the parameter estimation of the submodels for a particular multiple model structure. See (Murray-Smith and Johansen, 1997; Babuska, 1998; Gasso et al., 2001; Venkat et al., 2003; Orjuela et al., 2006) and the references therein for further information about these techniques.

\subsection{Takagi-Sugeno multiple model}

Takagi-Sugeno multiple model structure is conventionally employed in multiple model analysis and synthesis. This multiple model has the following structure:

$$
\begin{aligned}
& \dot{x}(t)=\left\{\sum_{i=1}^{L} \mu_{i}(\xi(t)) A_{i}\right\} x(t)+\left\{\sum_{i=1}^{L} \mu_{i}(\xi(t)) B_{i}\right\} u(t), \\
& y(t)=\left\{\sum_{i=1}^{L} \mu_{i}(\xi(t)) C_{i}\right\} x(t),
\end{aligned}
$$

where $x \in \mathbb{R}^{n}$ is the state vector, $u \in \mathbb{R}^{m}$ the input vector, $y \in \mathbb{R}^{p}$ the output vector and $A_{i} \in \mathbb{R}^{n \times n}$, $B_{i} \in \mathbb{R}^{n \times m}$ and $C_{i} \in \mathbb{R}^{p \times n}$ are known constant matrices. 
Moreover, the $\mu_{i}(\xi(t))$ are the weighting functions that ensure the transition between the contribution of each submodel. They have the following properties :

$$
\begin{aligned}
& \sum_{i=1}^{L} \mu_{i}(\xi(t))=1, \quad \forall t \\
& 0 \leq \mu_{i}(\xi(t)) \leq 1 \forall i=1 \ldots L, \forall t
\end{aligned}
$$

where $\xi(t)$ is the decision variable that depends on known quantities such the input or the output of the system.

It should be noted that the Takagi-Sugeno multiple model is similar to a system whose parameters vary with time. Indeed, from equation (1), one can see that the contribution of each submodel is taken into account through a blend between the parameters of the submodels.

\subsection{Decoupled multiple model}

Filev (1991) proposes another multiple model structure based on a parallel interconnection of the submodels. Here, this structure is slightly modified using a state representation as follows:

$$
\begin{aligned}
\dot{x}_{i}(t) & =A_{i} x_{i}(t)+B_{i} u(t), \\
y_{i}(t) & =C_{i} x_{i}(t) \\
y(t) & =\sum_{i=1}^{L} \mu_{i}(\xi(t)) y_{i}(t),
\end{aligned}
$$

where $x_{i} \in \mathbb{R}^{n_{i}}$ and $y_{i} \in \mathbb{R}^{p}$ are respectively the state vector and the output vector for the $\mathrm{i}^{\text {th }}$ submodel and where $A_{i} \in \mathbb{R}^{n_{i} \times n_{i}}, B_{i} \in \mathbb{R}^{n_{i} \times m}$ and $C_{i} \in \mathbb{R}^{p \times n_{i}}$ are the constant matrices.

In this multiple model structure the contribution of each submodel is taken into account by a blend between the outputs of the submodels via a weighted sum. It is obvious that the principal interest of this structure is the decoupling between the submodels. Indeed, in contrast to the Takagi-Sugeno multiple model, in the decoupled multiple model the dimension of the state vector $x_{i}$ of each submodel can be different (of course the dimension of the input and output vectors for all the submodels must be identical). Therefore, this structure is well adapted for modelling strongly nonlinear systems whose structure varies with the operating regime, for example when the complexity of the dynamic behaviour is not uniform in the operating range.

Notation: The following notations will be used throughout the paper: $P>0(P<0)$ denotes a positive (negative) definite matrix; $P^{T}$ is the transpose of the matrix P. I is the identity matrix of appropriated dimension. We shall simply write $\mu_{i}(\xi(t))=\mu_{i}(t)$ and $x(t-\tau(t))=x(\nabla)$ where $\tau(t)>0$ is a variable time-delay.

\section{PROBLEM FORMULATION}

The aim of this paper is to tackle the state estimation of a nonlinear system subject to delayed measurements. The nonlinear system is modelled by the following decoupled multiple model:

$$
\begin{aligned}
\dot{x}_{i}(t) & =A_{i} x_{i}(t)+B_{i} u(t) \\
y_{i}(t) & =C_{i} x_{i}(t) \\
y(t) & =\sum_{i=1}^{L} \mu_{i}(\xi(t-\tau(t))) y_{i}(t-\tau(t)) .
\end{aligned}
$$

The variable time-delay $\tau(t)$ is assumed perfectly known and satisfies the following conditions:

$$
\left\{\begin{array}{c}
0 \leq \tau(t) \leq \tau \\
\dot{\tau}(t) \leq \gamma<1
\end{array}\right.
$$

The state estimation of the multiple model (4) is achieved with the help of a proportional observer given by:

$$
\begin{aligned}
\dot{\hat{x}}_{i}(t) & =A_{i} \hat{x}_{i}(t)+B_{i} u(t)+K_{i}(y(t)-\hat{y}(t)), \\
\hat{y}_{i}(t) & =C_{i} \hat{x}_{i}(t) \\
\hat{y}(t) & =\sum_{i=1}^{L} \mu_{i}(\xi(t-\tau(t))) \hat{y}_{i}(t-\tau(t)),
\end{aligned}
$$

where $K_{i} \in \mathbb{R}^{n_{i} \times p}$ is the associated gain to $\mathrm{i}^{\text {th }}$ observer to be designed.

In this paper, the design of the observer consists in determining the matrices $K_{i}$ such that the estimation error given by:

$$
e_{i}(t)=x_{i}(t)-\hat{x}_{i}(t), \quad i=1 \ldots L
$$

converges towards zero in a finite time (exponential convergence) for any initial conditions. It is important to remark that the observer only uses the measurable signals i.e. the input and output of the system (the outputs of the submodels are not available). Therefore, it is necessary to take into account the blend between the outputs of the submodels, in the observer design stage, in order to ensure the convergence of the estimation error for an arbitrary blend between the submodels.

\section{MAIN RESULTS}

\subsection{Stability of the decoupled multiple model}

The use of an augmented state vector allows to rewrite the multiple model (4) in the following compact form: 


$$
\begin{aligned}
\dot{x}(t) & =\tilde{A} x(t)+\tilde{B} u(t), \\
y(t) & =\tilde{C}(\nabla) x(\nabla),
\end{aligned}
$$

where

$$
\begin{aligned}
\tilde{A} & =\left[\begin{array}{ccccc}
A_{1} & 0 & 0 & 0 & 0 \\
0 & \ddots & 0 & 0 & 0 \\
0 & 0 & A_{i} & 0 & 0 \\
0 & 0 & 0 & \ddots & 0 \\
0 & 0 & 0 & 0 & A_{L}
\end{array}\right], \tilde{B}=\left[\begin{array}{c}
B_{1} \\
\vdots \\
B_{i} \\
\vdots \\
B_{L}
\end{array}\right], \\
\tilde{C}(\nabla) & =\left[\mu_{1}(\nabla) C_{1} \cdots \mu_{i}(\nabla) C_{i} \cdots \mu_{L}(\nabla) C_{L}\right] \text { and } \\
x(t) & =\left[x_{1}(t) \cdots x_{i}(t) \cdots x_{L}(t)\right]^{T} \in \mathbb{R}^{n}, n=\sum_{i=1}^{L} n_{i} .
\end{aligned}
$$

The stability of a decoupled multiple model can be easily established by analysing the eigenvalues of the matrix $\tilde{A}$. Indeed, notice that the matrix $\tilde{A}$ is a block diagonal matrix. Therefore, all eigenvalues of this matrix are in the left-half complex plane if and only if all eigenvalues of every matrices $A_{i}$ are in the left-half complex plane.

To sum up, a decoupled multiple model is stable if and only if all submodels are stable, in contrast to the Takagi-Sugeno multiple model where the stability depends not only on the stability of the submodels but also on the weighting function values. In the sequel, the multiple model is assumed to be stable.

\subsection{Estimation error convergence}

The aim of this section is to investigate the state estimation of a decoupled multiple model in presence of delayed measurements. Here, the second Lyapunov method is employed in order to provide delay-independent sufficient conditions, in LMIs terms (Boyd et al., 1994), for ensuring the estimation error convergence.

In the following, we will investigate the exponential convergence of the estimation error. Indeed, the exponential convergence is a strong form of convergence that guarantees dynamic performances of the estimation error, in particular a convergence velocity via a decay rate.

Theorem 1. Consider the decoupled multiple model (4), the observer (6) and the variable timedelay (5). The exponential convergence of the estimation error is guaranteed if there exists two symmetric, positive definite matrices $P$ and $Q$, a matrix $G$ and a positive scalar $\alpha$ such that:

$$
\left[\begin{array}{cc}
(\tilde{A}+\alpha \mathrm{I})^{T} P+P(\tilde{A}+\alpha \mathrm{I})+Q & -G \tilde{C}_{i} \\
-\left(G \tilde{C}_{i}\right)^{T} & -(1-\gamma) e^{-2 \alpha \tau} Q
\end{array}\right]<0,
$$

for $i=1 \ldots L$ where $\tilde{C}_{i}=\left[\begin{array}{lllll}0 & \cdots & C_{i} & \cdots & 0\end{array}\right]$. The scalar $\alpha$ is called the decay rate and the observer gain is deduced from $\tilde{K}=P^{-1} G$.
Proof : The observer represented by equation (6) can be rewritten, in a compact form, using the block matrices (8):

$$
\begin{aligned}
\dot{\hat{x}}(t) & =\tilde{A} \hat{x}(t)+\tilde{B} u(t)+\tilde{K}(y(t)-\hat{y}(t)), \\
\hat{y}(t) & =\tilde{C}(\nabla) \hat{x}(\nabla),
\end{aligned}
$$

where

$$
\tilde{K}=\left[K_{1} \cdots K_{i} \cdots K_{L}\right]^{T}, \in \mathbb{R}^{n} .
$$

The dynamics of the estimation error is given by:

$$
\dot{e}(t)=\tilde{A} e(t)-\tilde{K} \tilde{C}(\nabla) e(\nabla) .
$$

The exponential convergence is investigated via a Lyapunov-Krasovskii functional proposed by Mondié and Kharitonov (2005):

$$
\begin{aligned}
V(t) & =e^{T}(t) P e(t) \\
& +\int_{-\tau(t)}^{0} e^{T}(t+\theta) e^{2 \alpha \theta} Q e(t+\theta) d \theta,
\end{aligned}
$$

where $P$ and $Q$ are symmetric, positive definite matrices. Let us notice that the exponential convergence is ensured if the two following conditions are guaranteed:

(1) $V(t)>0$,

(2) $\dot{V}(t)+2 \alpha V(t)<0$.

The derivative of the functional (12) with respect to time yields:

$$
\begin{aligned}
\dot{V}(t) & =\dot{e}^{T}(t) P e(t)+e^{T}(t) P \dot{e}(t)+e^{T}(t) Q e(t) \\
& -(1-\dot{\tau}(t)) e^{-2 \alpha \tau(t)} e^{T}(\nabla) Q e(\nabla) \\
& -2 \alpha \int_{-\tau(t)}^{0} e^{T}(t+\theta) e^{2 \alpha \theta} Q e(t+\theta) d \theta,
\end{aligned}
$$

that can be upper bounded using the time-delay conditions (5):

$$
\begin{aligned}
\dot{V}(t) & \leq \dot{e}^{T}(t) P e(t)+e^{T}(t) P \dot{e}(t) \\
& +e^{T}(t) Q e(t)-(1-\gamma) e^{-2 \alpha \tau} e^{T}(\nabla) Q e(\nabla) \\
& -2 \alpha \int_{-\tau(t)}^{0} e^{T}(t+\theta) e^{2 \alpha \theta} Q e(t+\theta) d \theta,
\end{aligned}
$$

that becomes by considering (11):

$$
\begin{aligned}
\dot{V}(t)= & {\left[\begin{array}{c}
e(t) \\
e(\nabla)
\end{array}\right]^{T}\left[\begin{array}{cc}
\tilde{A}^{T} P+P \tilde{A}+Q & -P \tilde{K} \tilde{C}(\nabla) \\
-(\tilde{K} \tilde{C}(\nabla))^{T} P & -(1-\gamma) e^{-2 \alpha \tau} Q
\end{array}\right]\left[\begin{array}{c}
e(t) \\
e(\nabla)
\end{array}\right] } \\
& -2 \alpha \int_{-\tau(t)}^{0} e^{T}(t+\theta) e^{2 \alpha \theta} Q e(t+\theta) d \theta
\end{aligned}
$$

Moreover, notice that the functional (12) can be rewritten as follows: 


$$
\begin{aligned}
V(t) & =\left[\begin{array}{c}
e(t) \\
e(\nabla)
\end{array}\right]^{T}\left[\begin{array}{ll}
P & 0 \\
0 & 0
\end{array}\right]\left[\begin{array}{c}
e(t) \\
e(\nabla)
\end{array}\right] \\
& +\int_{-\tau(t)}^{0} e^{T}(t+\theta) e^{-2 \alpha \theta} Q e(t+\theta) d \theta,
\end{aligned}
$$

hence the expression $\dot{V}+2 \alpha V$ yields:

$$
\begin{aligned}
\dot{V}+2 \alpha V & \leq\left[\begin{array}{c}
e(t) \\
e(\nabla)
\end{array}\right]^{T}\left\{\left[\begin{array}{cc}
\tilde{A}^{T} P+P \tilde{A}+Q & -P \tilde{K} \tilde{C}(\nabla) \\
-(\tilde{K} \tilde{C}(\nabla))^{T} P & -(1-\gamma) e^{-2 \alpha \tau} Q
\end{array}\right]\right. \\
& \left.+2 \alpha\left[\begin{array}{ll}
P & 0 \\
0 & 0
\end{array}\right]\right\}\left[\begin{array}{c}
e(t) \\
e(\nabla)
\end{array}\right] .
\end{aligned}
$$

The right hand of the above inequality is a quadratic form in $[e(t) e(\nabla)]^{T}$. The negativity of $\dot{V}(t)+2 \alpha V$ is therefore guaranteed if and only if:

$$
\left[\begin{array}{cc}
(\tilde{A}+\alpha \mathrm{I})^{T} P+P(\tilde{A}+\alpha \mathrm{I})+Q & -P \tilde{K} \tilde{C}(\nabla) \\
-(\tilde{K} \tilde{C}(\nabla))^{T} P & -(1-\gamma) e^{-2 \alpha \tau} Q
\end{array}\right]<0 .
$$

Moreover, it is important to notice that the matrix $\tilde{C}(\nabla)$ can be rewritten as follows:

$$
\tilde{C}(\nabla)=\sum_{i=1}^{L} \mu_{i}(\nabla) \tilde{C}_{i},
$$

where the constant, block matrix $\tilde{C}_{i}$ takes the following form:

$$
\tilde{C}_{i}=\left[\begin{array}{lllll}
0_{\left(p \times n_{1}\right)} & \cdots & C_{i} & \cdots & 0_{\left(p \times n_{L}\right)}
\end{array}\right] .
$$

By considering (19) and taking into account the property (2a) of the weighting functions, the matrix inequality (18) may be rewritten as:

$$
\sum_{i=1}^{L} \mu_{i}(\nabla)\left[\begin{array}{cc}
(\tilde{A}+\alpha \mathrm{I})^{T} P+P(\tilde{A}+\alpha \mathrm{I})+Q & -P \tilde{K} \tilde{C}_{i} \\
-\left(\tilde{K} \tilde{C}_{i}\right)^{T} P & -(1-\gamma) e^{-2 \alpha \tau} Q
\end{array}\right]<0 .
$$

According to property (2b), the above matrix inequality is also satisfied if:

$$
\left[\begin{array}{cc}
(\tilde{A}+\alpha \mathrm{I})^{T} P+P(\tilde{A}+\alpha \mathrm{I})+Q & -P \tilde{K} \tilde{C}_{i} \\
-\left(\tilde{K} \tilde{C}_{i}\right)^{T} P & -(1-\gamma) e^{-2 \alpha \tau} Q
\end{array}\right]<0,
$$

for $i=1$... $L$, that is a sufficient condition for ensuring the exponential convergence of the estimation error. Notice that these matrix inequalities are nonlinear (bilinear) in $\tilde{K}$ and $P$. Therefore, it is not possible to solve them directly using classical LMI tools. However, the change of variables $G=P \tilde{K}$ allows the linearisation of the matrix inequalities (21) as follows:

$$
\left[\begin{array}{cc}
(\tilde{A}+\alpha \mathrm{I})^{T} P+P(\tilde{A}+\alpha \mathrm{I})+Q & -G \tilde{C}_{i} \\
-\left(G \tilde{C}_{i}\right)^{T} & -(1-\gamma) e^{-2 \alpha \tau} Q
\end{array}\right]<0,
$$

for $i=1 \ldots L$, that is a LMI in $P, G$ and $Q$. Hence, the proof of Theorem 1 is completed.

\section{SIMULATION EXAMPLE}

Let us consider the state estimation of a decoupled multiple model with $L=2$ submodels. The parameters of the submodels are:

$$
\begin{aligned}
A_{1} & =\left[\begin{array}{ccc}
-2 & 0.5 & 0.6 \\
-0.3 & -0.9 & 0 \\
-1.3 & 0.6 & -0.8
\end{array}\right], & & A_{2}=\left[\begin{array}{cc}
-0.2 & -0.6 \\
0.3 & -1
\end{array}\right], \\
B_{1} & =\left[\begin{array}{lll}
1 & 0.2 & 0.5
\end{array}\right]^{T}, & B_{2} & =\left[\begin{array}{ll}
-0.5 & 0.8
\end{array}\right]^{T}, \\
C_{1} & =\left[\begin{array}{lll}
1 & 0.8 & 0.5
\end{array}\right], & C_{2} & =\left[\begin{array}{ll}
0.7 & 0.3
\end{array}\right] .
\end{aligned}
$$

Here, the decision variable $\xi(t)$ is the input signal $u(t) \in[0,1]$. The weighting functions are obtained from normalised Gaussian functions:

$$
\begin{aligned}
& \mu_{i}(\xi(t))=\omega_{i}(\xi(t)) / \sum_{j=1}^{L} \omega_{j}(\xi(t)), \\
& \omega_{i}(\xi(t))=\exp \left(-\left(\xi(t)-c_{i}\right)^{2} / \sigma^{2}\right),
\end{aligned}
$$

with the standard deviation $\sigma=0.4$ and the centres $c_{1}=0.3$ and $c_{2}=0.7$. The eigenvalues of the matrix $\tilde{A}$ lie in the open left-half plane:

$$
\lambda=[-1.33 \pm 0.71 i,-1.03,-0.6 \pm 0.14 i],
$$

therefore the multiple model is stable.

The time-delay that appears in the output of the systems has the form: $\tau(t)=0.5+0.45 \sin (0.5 t)$. The upper bound of its derivative is given by: $\gamma=0.225$.

A solution that satisfied the Theorem 1, is given by:

$$
\begin{aligned}
& P=\left[\begin{array}{ccccc}
1.396 & -1.598 & -0.548 & -0.139 & -1.302 \\
-1.598 & 8.306 & 3.216 & -0.014 & -0.472 \\
-0.548 & 3.216 & 1.591 & 0.066 & 0.517 \\
-0.139 & -0.014 & 0.066 & 5.051 & -1.317 \\
-1.302 & -0.472 & 0.517 & -1.317 & 9.350
\end{array}\right], \\
& Q=\left[\begin{array}{ccccc}
1.907 & 2.193 & 1.207 & 0.102 & -1.278 \\
2.193 & 4.327 & 2.158 & 0.340 & -3.186 \\
1.207 & 2.158 & 1.118 & 0.168 & -1.476 \\
0.102 & 0.340 & 0.168 & 0.718 & -0.137 \\
-1.278 & -3.186 & -1.476 & -0.137 & 3.312
\end{array}\right], \\
& \tilde{K}=\left[\begin{array}{lllll}
0.9330 & 0.9017 & -1.5429 & 0.1059 & 0.1568
\end{array}\right]^{T} \text {, }
\end{aligned}
$$

with a decay rate $\alpha=0.2$.

In the simulation an uniformly distributed random signal, generated by Simulink, is added to the output. The obtained estimation error is plotted in figure 1 (top and center), the measured and the estimated output is shown at the bottom of figure 1 . The error around the origin time is due to the differences between initial conditions of the multiple model and the observer. The suggested observer provides a good state estimation under measurement noise. 

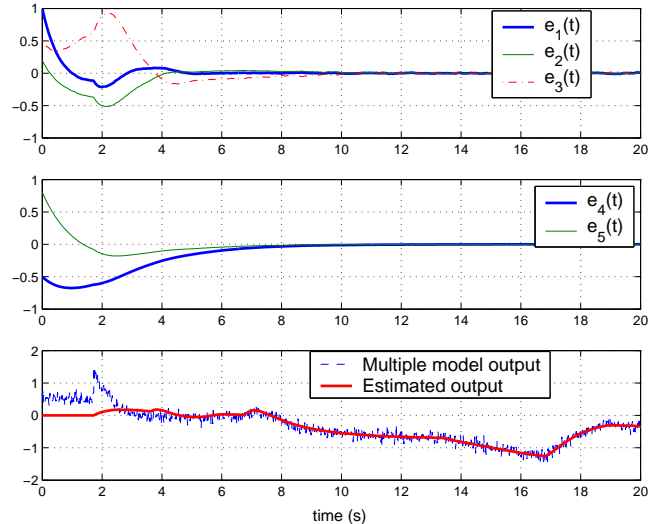

Fig. 1. Estimation error (top and center), mesured and estimated output (bottom)

\section{CONCLUSION}

The state estimation of a nonlinear system in presence of delayed measurements has been investigated in this communication. The state observer is built by modelling the nonlinear system by a decoupled multiple model. In contrast to the well known Takagi-Sugeno observer, in the proposed observer each submodel has its own state vector that can have a different dimension. The exponential convergence of the estimation error is ensured if a set of delay-independent sufficient conditions are satisfied. Encouraging results are illustrated through a simulation example.

In a future work, the proposed approach will be extended in order to take into account a parametric uncertain multiple model. An interesting prospect can be to consider in particular an uncertain time-delay i.e. the exact value of the timedelay in the observer is not precisely known.

\section{ACNOWELEDGEMENTS}

This research was supported by the TASSILI ${ }^{\circ} 07$ program under MDU grant 714.

\section{REFERENCES}

Babuska, R. (1998). Fuzzy modeling for control. Kluwer Academic Pulishers. Boston.

Boyd, S., L. El Ghaoui, E. Feron and V. Balakrishnan (1994). Linear Matrix Inequalities in System and Control Theory. SIAM studies in applied mathematics. Philadelphia, P.A.

Cao, Young-Yan. and P.M. Frank (2001). Stability analysis and synthesis of nonlinear time-delay systems via linear Takagi-Sugeno fuzzy models. Fuzzy Sets and Systems 124(2), 213-229.

Chen, C.W., W.L. Chiang and F.H. Hsiao (2004). Stability analysis of T-S fuzzy models for nonlinear multiple time-delay interconnected systems. Mathematics and Computers in Simulation 66(6), 523-537.

Filev, D. (1991). Fuzzy modeling of complex systems. International Journal of Approximate Reasoning 5(3), 281-290.
Fridman, E., U. Shaked and L. Xie (2003). Robust Hinf filtering of linear systems with timevarying delay. IEEE Transactions on Automatic Control 48(1), 159-165.

Gasso, K., G. Mourot and J. Ragot (2001). Structure identification in multiple model representation: elimination and merging of local models. In: 40th IEEE Conference on Decision and Control. Orlando, USA. pp. 29922997.

Gawthrop, P.J. (1995). Continuous-time local state local model networks. In: IEEE Conference on Systems, Man \& Cybernetics. Vancouver, Canada. pp. 852-857.

Germani, A., C. Manes and P. Pepe (2002). A new approach to state observation of nonlinear systems with delayed output. IEEE Transactions on Automatic Control 47(1), 96-101.

Gregorcic, G. and G. Lightbody (2000). Control of highly nonlinear processes using self-tuning control and multiple/local model approaches. In: 2000 IEEE International Conference on Intelligent Engineering Systems, INES 2000. Portoroz, Slovenie. pp. 167-171.

Jiang, X., W. Xu and Q.L. Han (2005). Observerbased fuzzy control dessign with adaptation to delay parameter for time-delay systems. Fuzzy Sets and Systems 152(3), 637-649.

Kazantzis, N. and R. Wright (2005). Nonlinear observer design in the presence of delayed output measurements. Systems $\&$ Control Letters 54(9), 877-886.

Mondié, S. and V. L. Kharitonov (2005). Exponential estimates for retarded time-delay systems: An LMI approach. IEEE Transactions on Automatic Control 50(2), 268-273.

Murray-Smith, R. and T.A. Johansen (1997). Multiple model approaches to modelling and control. Taylor \& Francis. London.

Orjuela, R., D. Maquin and J. Ragot (2006). Nonlinear system identification using uncoupled state multiple-model approach. In: Workshop on Advanced Control and Diagnosis, $A C D$ '2006. Nancy, France.

Richard, J. P. (2003). Time-delay systems: an overview of some recent advances and open problems. Automatica 39(10), 1667-1694.

Sename, O. (2001). New trends in design of observers for time-delay systems. Kybernetika 37(4), 427-458.

Venkat, A.N., P. Vijaysai and R.D. Gudi (2003). Identification of complex nonlinear processes based on fuzzy decomposition of the steady state space. Journal of Process Control 13(6), 473-488.

Yang, H. and M. Saif (1998). Observer design and fault diagnosis for state-retarded dynamical systems. Automatica 34(2), 217-227. 